\title{
AN APPROXIMATION IN SYMMETRY CONSERVING CONFIGURATION MIXING CALCULATIONS*
}

\author{
M. Borrajo, J.L. Egido \\ Departamento de Física Teórica, Universidad Autónoma de Madrid \\ 28049 Madrid, Spain \\ (Received November 10, 2015) \\ An approximation is proposed in Beyond mean field calculations to re- \\ duce the size of the grid subtended by the generator coordinates by one \\ order of magnitude. We show the quality of the approximation calculat- \\ ing the excitation energies of the titanium isotopes and the E2 transition
} probabilities.

DOI:10.5506/APhysPolBSupp.8.567

PACS numbers: 21.10.-k, 23.20.Lv, 21.10.Re, 21.60.Ev

\section{Introduction}

The basic approach to any many body theory is the Mean Field Approach (MFA), either by itself or as basis for more sophisticated theories. The most general MFA is the Hartree-Fock-Bogoliubov (HFB) theory which considers on equal footing the long and the short range correlations [1]. The HFB combined with effective interactions is well-known to provide a good description of bulk nuclear properties like binding energies, quadrupole moments etc. [2]. The incorporation of Beyond Mean Field (BMF) techniques such as the angular momentum (AM) and particle number (PN) projection, together with the consideration of fluctuations around the most probable values by the Generator Coordinate Method (GCM) has opened the door to the description of spectroscopic properties with effective forces such as the Skyrme [3], the Gogny [4] or relativistic ones [5]. The complexity of the triaxial angular momentum projection and the necessity of considering several generator coordinates has increased the CPU time necessary for these calculations so much that some approximations are needed to study heavy nuclei. In this work, we propose an approach that reduces the CPU time considerably and, at the same time, provides good results. In all calculations, we use the Gogny interaction [6].

\footnotetext{
* Presented at the XXII Nuclear Physics Workshop "Marie and Pierre Curie", Kazimierz
} Dolny, Poland, September 22-27, 2015. 


\section{Theory and numerical applications}

The HFB wave function (w.f.) is given by $|\phi\rangle=\Pi_{k} \alpha_{k}|-\rangle$, with the quasiparticle operators defined by the general Bogoliubov transformations

$$
\alpha_{\mu}=\sum_{k} U_{i \mu}^{*} c_{k}+V_{i \mu}^{*} c_{k}^{\dagger},
$$

with $c_{k}^{\dagger}, c_{k}$ the particle creation and annihilation operators in the original basis and $U$ and $V$ the Bogoliubov w.f. to be determined by the Ritz variational principle. Since the Bogoliubov transformation mixes creator and annihilator operators, the HFB w.f. is not an eigenstate of the particle number and one should keep the right number of particles at least on the average in the minimization process, i.e.,

$$
\delta E^{\prime}[\phi\{U, V\}]=0
$$

with

$$
E^{\prime}=\langle\phi|\hat{H}| \phi\rangle-\lambda_{N}\langle\phi|\hat{N}| \phi\rangle
$$

and the Lagrange multiplier $\lambda_{N}$ given by the constraint $\langle\phi|\hat{N}| \phi\rangle=N$. We know, however, that atomic nuclei do have good quantum numbers such as angular momentum, particle number and parity and that in order to calculate some observables, these quantum numbers are needed. A convenient approach is the so-called symmetry conserving mean field approximation (SCMFA). In this approach, the (intrinsic) w.f. is still a product w.f. but the quantum numbers are singled out by means of projectors, e.g., the w.f.

$$
\left|\psi_{M}^{N, I, \sigma}\right\rangle=\sum_{K} g_{K}^{\sigma} P_{M K}^{I} P^{N}|\phi\rangle
$$

with $P^{N}$ and $P_{M K}^{I}$ projectors on the particle number $(\mathrm{PN})$ and the angular momentum (AM), respectively, is an eigenstate of the particle number and the angular momentum operators. The index $\sigma$ labels the different states with the same $I$. The w.f. (4) depends only on the matrices $U$ and $V$ of the Bogoliubov transformation. The proper way to determine them is by the variational principle, i.e., by minimization of the projected energy

$$
\delta E^{N, I}[U, V]=\delta \frac{\left\langle\psi^{N, I}|\hat{H}| \psi^{N, I}\right\rangle}{\left\langle\psi^{N, I} \mid \psi^{N, I}\right\rangle}=0 .
$$

This is known as the variation after projection (VAP) approach and is the best way of finding the Bogoliubov matrices since only states with the right quantum numbers are considered in the variation. Sometimes the w.f. $\left|\psi^{N, I}\right\rangle$ 
is determined in the projection after variation (PAV) approach. In this case, the Bogoliubov matrices are determined by minimization of the unprojected energy, Eq. (3), and afterwards the projection takes place. Manifestly the PAV approach is worse than the VAP one. In the case of the PN, the worst situation occurs in the weak pairing regime where the HFB w.f. collapses to the HF one and the superfluid phase is missed. The solution of Eq. (5) with $\mathrm{PN}$ and AMP is very complicated because of the non-linear character of the HFB equations and the large CPU time needed for the AMP. Fortunately, the more necessary PN-VAP is feasible and thus the pairing collapse avoided.

The w.f. $\left|\psi_{M}^{N, I, \sigma}\right\rangle$ behaves in many ways like a product w.f., therefore, in order to describe the shape coexistence or configuration mixing, fluctuations must be included. This has led to the modern beyond mean field calculations with the GCM inspired Ansatz [2]

$$
\left|\Psi_{\sigma}^{N, I}\right\rangle=\sum_{\vec{\alpha}} f_{\sigma}^{I}(\vec{\alpha}) P^{N} P_{M K}^{I}|\phi(\vec{\alpha})\rangle=\sum_{\vec{\alpha}} f_{\sigma}^{I}(\vec{\alpha})\left|\Phi^{N, I}(\vec{\alpha})\right\rangle
$$

where we have introduced $\left|\Phi^{N, I}(\vec{\alpha})\right\rangle$ and $\vec{\alpha}$ denotes the relevant degrees of freedom to be used as coordinates to generate a set of w.f. $\phi(\vec{\alpha})$ and the index $K$, for example $\vec{\alpha}=(\beta, \gamma, K)$. The weights $f_{\sigma}^{I}(\vec{\alpha})$ are to be determined by the variational principle which leads to the Hill-Wheeler (HW) equation.

The choice of the coordinates $\vec{\alpha}$ is a very crucial issue. The simplest approach is to consider axially symmetric calculations. In this case, there is only one coordinate, namely the $\beta$ deformation and $K=0$. There have been calculations of this type with the Skyrme [2], Gogny [8,10] and relativistic [9] interactions. This approach provides a good qualitative description of nuclei close to axial symmetry. The main drawbacks are obviously the absence of triaxial effects and the prediction of very stretched spectra. A considerably more realistic case is to consider the triaxial deformation. In this case, $\vec{\alpha}=(\beta, \gamma, K)$ and again there have been calculations with the three effective interactions mentioned above [3-5]. In these calculations, the $\gamma$ bands are properly described and the spectra are less stretched than in the axial case but still stretched as compared with the experiment. As an example of the mentioned approaches, we display in Fig. 1 (taken from Ref. [4]) the spectrum of ${ }^{24} \mathrm{Mg}$ compared with the experiment. In the axial case (left), the experimental ground state band is well reproduced, a well developed $\beta$-band is found though at too high excitation energy, but the $\gamma$-band is not found. If we now include triaxial effects, i.e., the $\gamma$ degree of freedom, we obtain the triaxial spectrum shown in the middle panel of Fig. 1. Now, a well developed $\gamma$-band is found and a considerable lowering of the $\beta$-band is obtained. 


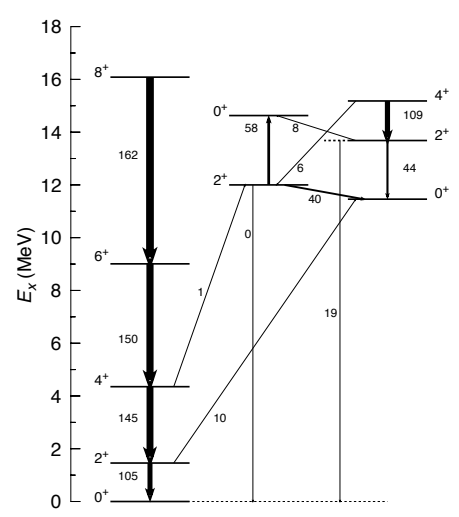

Axial

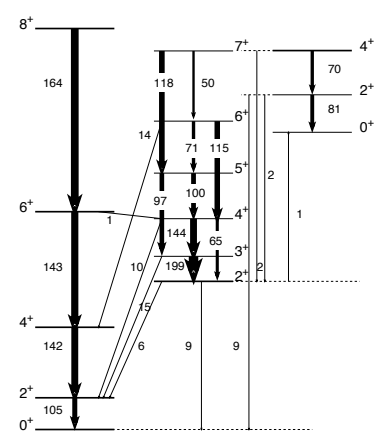

Triaxial

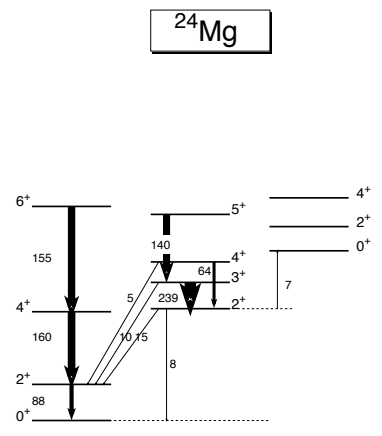

Experiment

Fig. 1. Calculated excitation energies and reduced transition probabilities $B(\mathrm{E} 2)$ (in $e^{2} \mathrm{fm}^{4}$ ) in ${ }^{24} \mathrm{Mg}$ obtained using axially symmetric (left) and triaxial (middle) GCM-PNAMP approaches compared to the experimental values (right). The experimental values are taken from [11].

The issue of the stretched spectra is general $[9,10]$ and it can be easily understood. As mentioned above, an AM-VAP solution of the symmetry conserving HFB equation (5) is very CPU time demanding and with effective interactions and large configurations space not feasible. That means the AMP is performed after the variation and the angular momentum is completely ignored in the determination of the HFB w.f. by the variational principle. To remedy this situation, we have recently proposed to incorporate the cranking frequency as a generator coordinate [12-14]. The Ansatz of Eq. (6) now looks like

$$
\left|\Psi_{\sigma}^{N, I}\right\rangle=\sum_{\beta, \gamma, \omega, K} f_{\sigma}^{I}(\beta, \gamma, \omega, K) P^{N} P_{M K}^{I}|\phi(\beta, \gamma, \omega)\rangle .
$$

The HFB w.f. $|\phi(\beta, \gamma, \omega)\rangle$ are determined by minimizing [15] the energy functional

$$
E[\phi]=\frac{\left\langle\phi\left|H P^{Z} P^{N}\right| \phi\right\rangle}{\left\langle\phi\left|P^{Z} P^{N}\right| \phi\right\rangle}-\left\langle\phi\left|\omega \hat{J}_{x}+\lambda_{q_{0}} \hat{Q}_{20}+\lambda_{q_{2}} \hat{Q}_{22}\right| \phi\right\rangle,
$$

where $\hat{Q}_{2 \mu}$ and $\hat{J}_{x}$ are quadrupole moment and the $x$-component of the angular momentum operators, respectively, $\lambda_{q_{0}}$ and $\lambda_{q_{2}}$ the Lagrange multipliers determined by the constraints $\left\langle\phi\left|\hat{Q}_{20}\right| \phi\right\rangle=q_{20}$ and $\left\langle\phi\left|\hat{Q}_{22}\right| \phi\right\rangle=q_{22}$, while $\omega$ is kept constant during the minimization process. $(\beta, \gamma)$ are defined by $\beta=\sqrt{20 \pi\left(q_{20}^{2}+2 q_{22}^{2}\right)} / 3 r_{0}^{2} A^{5 / 3}, \gamma=\arctan \left(\sqrt{2} q_{22} / q_{20}\right)$ with $r_{0}=1.2 \mathrm{fm}$ and $A$ is the mass number. That means, the HFB w.f. are determined 
in the PN-VAP approach [16]. Interestingly, the incorporation of $\omega$ in the GCM Ansatz of Eq. (7) is a generalization of the double projection method of Peierls and Thouless [17, 18] for the case of rotations. This method is known to provide the exact translational mass in the case of translations. We, therefore, expect that the moments of inertia of our bands will be close to the ones of the AM-VAP providing the sought-after spectrum compression. In the light of Eq. (8), one can understand the origin of the stretching. In the spirit of the cranking model, a state with angular momentum $I$ can be obtained semi-classically with the constraint $\left\langle\phi\left|\hat{J}_{x}\right| \phi\right\rangle=\sqrt{I(I+1)}$. Therefore, if the variation is performed without $\left\langle\hat{J}_{x}\right\rangle$ constraint, as in the former calculations, then $\left\langle\phi\left|\hat{J}_{x}\right| \phi\right\rangle=0$ and the variational principle provides the w.f. optimal for $I=0$. The states with $I \neq 0$ are clearly disfavored as compared with $I=0$ and the larger the $I$, the higher the state is disfavored thus providing a stretched spectrum.

Of course, one could argue that the constrained variational principle of Ritz used to determine the intrinsic basis states is very effective in determining the wave function of the ground state with the given quantum numbers and constraints. Ground states of the SCCM calculations benefit from this fact, however, SCCM excited states with the same or different quantum numbers are not favored by it and depend more strongly on the basis size (number of generator coordinates). Consequently, in restricted self-consistent calculations, a stretched spectrum is expected which will be squeezed by an appropriate increase of the basis size, for example by allowing pairing fluctuations. Indeed, since the monopole (pairing) and the quadrupole (deformation) are the most relevant degrees of freedom, it seems reasonable to consider both of them on an equal footing. Recently in Ref. [19,20], the effect of including fluctuations of the pairing gap in the GCM Ansatz in realistic calculations has been investigated. In these calculations, a given compression of the spectra is found but a comparison with the experiment shows that it is not enough. However, if one includes the angular frequency as a generator coordinate as in the calculations performed in [12-14], one obtains the needed compression and a good agreement with the experiment. This means that the alignments induced by the cranking frequency are independent of the effects produced by the pairing fluctuations.

The consideration of the angular frequency as a generator coordinate has a big impact on the CPU time of the calculations because the cranking term $-\omega \hat{J}_{x}$ added in Eq. (8) causes a time reversal symmetry breaking (TRSB). Besides the obvious fact of adding one more coordinate to the calculations, the symmetry breaking has two important consequences: First, one cannot perform axially symmetric calculations even in nuclei where no triaxial effects are expected and second, the usual $0^{\circ} \leq \gamma \leq 60^{\circ}$ sextant is not equivalent anymore to all sextants in the $\{\beta, \gamma\}$ plane and the half plane 
$-60^{\circ} \leq \gamma \leq 120^{\circ}$ must be used. In general, the consideration of $\hbar \omega$ as a generator coordinate amounts to an increase of two orders of magnitude in the CPU time.

In the calculations of Refs. [12-14], only light nuclei were studied and a small number of oscillator shells were considered as configuration space. In order to study heavier nuclei, some approximations are needed. In the SCCM Ansatz, Eq. (6), and for not too large angular momenta, it is sufficient to consider two or three $\omega$ values. For $\beta$, in general, about 12 mesh-points are needed and for $\gamma$ on the average, about 18 points. The largest energy dependence is with the $\beta$ degree of freedom but the coordinate that most increases the CPU time is the $\gamma$. The approximation that we investigate in this work is to perform triaxial calculations but without constraining on $\gamma$. That means, for fixed $\omega$ and $\beta$ values, Eq. (8) is solved (obviously without the constraint on $\hat{Q}_{22}$ ) and the corresponding self-consistent $\gamma$ value is determined by the variational principle. For a given $\beta$ and different $\omega$, in general, we obtain different $\gamma$ values increasing thereby the diversity in the mixing. To test the approach, we have performed calculations for the nucleus ${ }_{22} \mathrm{Ti}$, which we already studied in Ref. [10] in an axially symmetric approach. The configuration space has eight oscillator shells. Since we are only interested in the low-spin region, we consider only two $\hbar \omega$ values, namely $\hbar \omega=0.0$ $\mathrm{MeV}$ and $\hbar \omega=0.5 \mathrm{MeV}$. We use the interval $0 \leq \beta \leq 0.6$ with a step size of 0.05 , i.e., 13 points for $\hbar \omega=0.0 \mathrm{MeV}$ and 12 points for $\hbar \omega=0.5 \mathrm{MeV}$. That means, we have to solve a Hill-Wheeler equation with 25 points and triaxial angular projection. In Fig. 2, we show the excitation energies of the $2_{1}^{+}$states

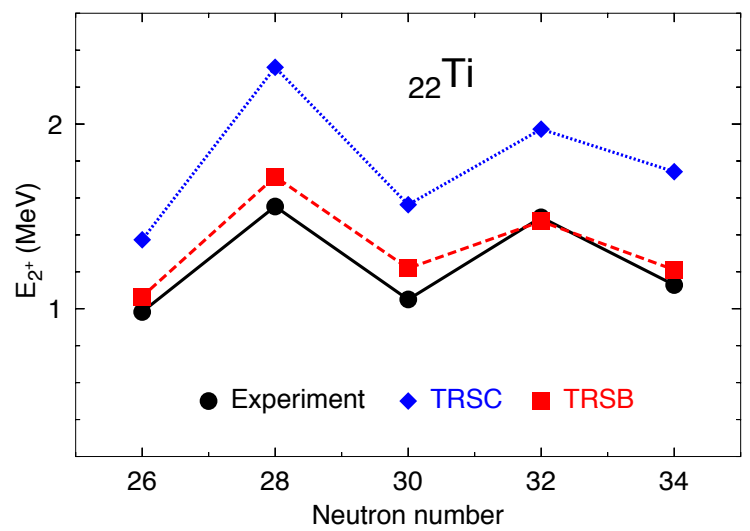

Fig. 2. (Color on-line) Excitation energies of the $2_{1}^{+}$states in the titanium isotopes in two approaches: Time reversal symmetry conserving (filled diamonds, blue) and time reversal symmetry breaking (filled squares, red). The experimental values [21-24] (bullets, black) are also shown. 
for the titanium isotopes in two approximations in comparison to the experimental results. The simplest approach is assuming axial symmetry, i.e., in the calculations only $\hbar \omega=0.0$ and $13 \beta$ points are considered, these are time reversal symmetry conserving calculations (TRSC). As compared with the experiment, these calculations provide the right behavior of the energy for the different isotopes but with too large values. In the second calculation, we add the 12 points corresponding to $\hbar \omega=0.5 \mathrm{MeV}$, these are TRSB calculations and a triaxial angular momentum projection must be performed. As we can observe in Fig. 2, the energy lowering is very significant bringing the theoretical results almost in agreement with the experimental ones.

Another aspect of the SCCM calculations, not mentioned yet, which causes some trouble is that, in general, they provide larger collectivity than experimentally observed. In Fig. 3, we show the $B\left(\mathrm{E} 2 ; 0_{1}^{+} \longrightarrow 2_{1}^{+}\right)$values for the titanium isotopes in the same two approximations as before. The TRSC calculations provide $B(\mathrm{E} 2)$ values that are too high as compared with the experiment. The TRSB, however, decreases these values considerably so a very good agreement is obtained.

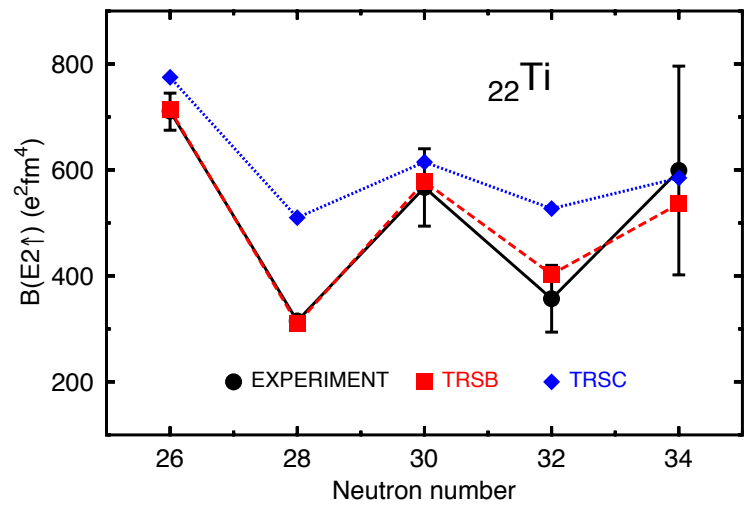

Fig. 3. (Color on-line) $B\left(\mathrm{E} 2 ; 0_{1}^{+} \longrightarrow 2_{1}^{+}\right)$transition probabilities in the titanium isotopes in two approaches: Time reversal symmetry conserving (filled diamonds, blue) and time reversal symmetry breaking (filled squares, red). The experimental values [25] (bullets, black) are also shown.

In conclusion, we have presented an approach to time reversal symmetry breaking calculations that reduce considerably the computational burden. This approximation will allow to extend the TRSB calculations to medium and heavy nuclei allowing an accurate description of nuclear properties. 
The authors gratefully thank T.R. Rodríguez for discussions. This work was supported by the Spanish Ministerio de Economía y Competitividad under contracts FPA2011-29854-C04-04, FPA2014-57196-C5-2-P and BES2012-059405.

\section{REFERENCES}

[1] P. Ring, P. Schuck, The Nuclear Many-body Problem, Springer-Verlag, Berlin 1980.

[2] M. Bender, P.-H. Heenen, P.-G. Reinhard, Rev. Mod. Phys. 75, 121 (2003).

[3] M. Bender, P.-H. Heenen, Phys. Rev. C 78, 024309 (2008).

[4] T.R. Rodríguez, J.L. Egido, Phys. Rev. C 81, 064323 (2010).

[5] J.M. Yao et al., Phys. Rev. C 81, 044311 (2010).

[6] J.F. Berger, M. Girod, D. Gogny, Nucl. Phys. A 428, 23c (1984).

[7] D. Hill, J.A. Wheeler, Phys. Rev. 89, 1102 (1953).

[8] R. Rodriguez-Guzman, J.L. Egido, L.M. Robledo, Nucl. Phys. A 709, 201 (2002).

[9] T. Nikšić, D. Vretenar, P. Ring, Phys. Rev. C 74, 064309 (2006).

[10] T.R. Rodríguez, J.L. Egido, Phys. Rev. Lett. 99, 062501 (2007).

[11] Evaluated Nuclear Structure Data File, http://www.nndc.bnl.gov/ensdf/

[12] M. Borrajo, T.R. Rodríguez, J.L. Egido, Phys. Lett. B 746, 341 (2015).

[13] J.L. Egido, M. Borrajo, T.R. Rodríguez, "Collective and Single-particle Motion in Beyond Mean Field Approaches", submitted for publication to Phys. Rev. Lett.

[14] T.R. Rodríguez, M. Borrajo, J.L. Egido, "Time Reversal Symmetry Breaking Effects in Beyond Mean Field Approaches", in preparation.

[15] J.L. Egido, J. Lessing, V. Martin, L.M. Robledo, Nucl. Phys. A 594, 70 (1995).

[16] M. Anguiano, J.L. Egido, L. M. Robledo, Nucl. Phys. A 696, 467 (2001).

[17] R.E. Peierls, D.T. Thouless, Nucl. Phys. 38, 154 (1962).

[18] J.L. Egido, Phys. Rev. C 27, 453 (1983).

[19] N. Lopez Vaquero et al., Phys. Rev. C 88, 064311 (2013).

[20] N. Lopez Vaquero et al., Phys. Lett. B 704, 520 (2011).

[21] S.N. Liddick et al., Phys. Rev. Lett. 92, 072502 (2004).

[22] R.V.F. Janssens et al., Phys. Lett. B 546, 55 (2002).

[23] S.N. Liddick et al., Phys. Rev. C 70, 064303 (2004).

[24] B. Fornal et al., Phys. Rev. C 70, 064304 (2004).

[25] D.-C. Dinca et al., Phys. Rev. C 71, 041302 (2005). 